\title{
ADP-Ribosyl Cyclases Regulate Early Development of the Sea Urchin
}

\author{
Latha Ramakrishnan ${ }^{1}$, Kevin Uhlinger ${ }^{2}$, Leslie Dale ${ }^{1}$, Amro Hamdoun², and Sandip Patel ${ }^{1, *}$ \\ ${ }^{1}$ Department of Cell and Developmental Biology, University College London, London WC1E 6BT, UK \\ ${ }^{2}$ Scripps Institution of Oceanography, University of California, San Diego, La Jolla, CA 92093-0202, USA
}

\begin{abstract}
ADP-ribosyl cyclases are multifunctional enzymes involved in the metabolism of nucleotide derivatives necessary for $\mathrm{Ca}^{2+}$ signalling such as CADPR and NAADP. Although $\mathrm{Ca}^{2+}$ signalling is a critical regulator of early development, little is known of the role of ADP-ribosyl cyclases during embryogenesis. Here we analyze the expression, activity and function of ADP-ribosyl cyclases in the embryo of the sea urchin-a key organism for study of both $\mathrm{Ca}^{2+}$ signalling and embryonic development. ADP-ribosyl cyclase isoforms (SpARC1-4) showed unique changes in expression during early development. These changes were associated with an increase in the ratio of CADPR:NAADP production. Over-expression of SpARC4 (a preferential cyclase) disrupted gastrulation. Our data highlight the importance of ADP-ribosyl cyclases during embryogenesis.
\end{abstract}

Keywords: ADP-Ribosyl Cyclase, Cyclic ADP-Ribose, NAADP, Early Development, Gastrulation.

\section{INTRODUCTION}

Changes in the concentration of cytosolic $\mathrm{Ca}^{2+}$ ions constitute a widespread and evolutionary conserved signalling pathway (Berridge et al., 2000). These signals regulate diverse cellular processes ranging from cell division to cell death (Berridge et al., 2000). It is perhaps not surprising then that $\mathrm{Ca}^{2+}$ plays a central role during early development (Slusarski and Pelegri, 2007; Whitaker, 2006). For example $\mathrm{Ca}^{2+}$ is necessary and sufficient for egg activation at fertilization (Whitaker, 2006) and is involved in later processes such as axis determination (Kume et al., 1997), convergent extension (Wallingford et al., 2001) and neural induction (Moreau et al., 2008). $\mathrm{Ca}^{2+}$ signals regulate a multitude of events including those during embryogenesis.

A major mechanism for generating $\mathrm{Ca}^{2+}$ signals is through the production of $\mathrm{Ca}^{2+}$ mobilizing messengers that release $\mathrm{Ca}^{2+}$ from intracellular $\mathrm{Ca}^{2+}$ stores (Berridge et al., 2000). Cyclic ADP-ribose (cADPR) and nicotinic acid adenine dinucleotide phosphate (NAADP) are two such messengers that target the ER and acidic $\mathrm{Ca}^{2+}$ stores (such as lysosomes), respectively (Lee, 2012; Patel and Docampo, 2010). Interestingly, despite their different mechanisms of action, both are synthesized by the same family of enzymes, the ADP-ribosyl cyclases via cyclisation and base-exchange reactions (Lee, 2000; Malavasi et al., 2008). This family includes CD38 a well

\footnotetext{
*Author to whom correspondence should be addressed.

E-mail: patel.s@ucl.ac.uk
}

characterized cell surface antigen (Howard et al., 1993). Indeed, all identified ADP-ribosyl cyclases appear to have exo-cytoplasmic catalytic sites suggesting compartmentalized messenger synthesis (De Flora et al., 2004) although their topology might not necessarily be fixed (Zhao et al., 2012). In addition to synthesis, ADP-ribosyl cyclases can also metabolize cADPR (and NAD) to ADP-ribose to regulate $\mathrm{Ca}^{2+}$ influx (Perraud et al., 2001) and NAADP to ADP-ribose phosphate (Graeff et al., 2006) which together with dephosphorylation (Berridge et al., 2002; Schmid et al., 2012) may serve to terminate NAADP-mediated $\mathrm{Ca}^{2+}$ signals. ADP-ribosyl cyclases are important multifunctional $\mathrm{Ca}^{2+}$ signalling enzymes.

ADP-ribosyl cyclase activity was first inferred in sea urchin egg homogenates over three decades ago (Clapper et al., 1987). As a basal deuterosotome, the sea urchin holds an important position in animal evolution and has been extensively used by both $\mathrm{Ca}^{2+}$ signallers and developmental biologists. But only relatively recently have molecular correlates of ADP-ribosyl cyclase activity been identified in this model organism. Four isoforms have been described in Stronglylocentrotus purpuratus (SpARC1-4) (Churamani et al., 2007; Ramakrishnan et al., 2010). SpARC1, 2 and 3 were independently cloned and referred to as $\operatorname{SpARC} \beta, \alpha$ and $\gamma$, respectively (Davis et al., 2008). SpARC1/ $\beta$ is unusual in that it is intracellular although it precise subcellular location (cortical granules in eggs $\mathrm{v}$ the ER) is unclear (Churamani et al., 2007; Davis et al., 2008). SpARCs show differences in catalytic activity with respect to their ability to metabolize the NAD surrogate NGD 
and the relative rates of cADPR versus NAADP production (Churamani et al., 2008; Ramakrishnan et al., 2010). SpARC2 for example preferentially produces NAADP over cADPR whereas SpARC4 preferentially produces cADPR over NAADP when assayed in parallel. These differences are likely attributable to a single residue within the active site because point mutation can convert the preference of SpARC4 to that of SpARC2 (Ramakrishnan et al., 2010). An ADP-ribosyl cyclase has recently been cloned from the sea star (Ramos et al., 2014) but its catalytic properties were not characterized and its relationship to other ADP-ribosyl cyclases is not clear at present. Our molecular understanding of these enzymes is advancing.

ADP-ribosyl cyclases play established roles in adult tissues which range from immune responses (PartidaSanchez et al., 2001) to social behaviour (Jin et al., 2007). We know little however about the part played by ADPribosyl cyclases during development. We showed that CD38 is critical for development of Xenopus laevis identifying a specific role for ADP-ribosyl cyclase in elongation of the anterior-posterior axis and differentiation of skeletal muscle (Churamani et al., 2012). Like mammals, Xenopus possess two ADP-ribosyl cyclases (CD38 and Bst1/CD157). Here we begin to define the role of ADPribosyl cyclases in development of the sea urchin, which possess an expanded ADP-ribosyl cyclase gene portfolio (Churamani et al., 2007; Ramakrishnan et al., 2010).

\section{MATERIALS AND METHODS}

Embryology. Stronglyocentrotus purpuratus eggs were fertilized and embryos grown as $0.5 \%(\mathrm{v} / \mathrm{v})$ suspensions in filtered sea water (FSW) at $16{ }^{\circ} \mathrm{C}$. The embryos were then collected at various time points (egg, 2, 3, 10, 21, 27.5, 33.5, 46, 58 and $63 \mathrm{~h}$ post-fertilization) according to standard protocols (Ettensohn et al., 2004). For RT PCR and activity measurements, the suspensions were gently centrifuged, the FSW aspirated and the pellets $(\sim 250 \mu \mathrm{L}$ packed volume) flash frozen and stored at $-80{ }^{\circ} \mathrm{C}$ prior to use.

Semi-quantitative RT-PCR. Total RNA was isolated from the frozen egg/embryo samples using the RNeasy kit (Qiagen), according to manufacturer's instructions. The samples were treated with on-column DNase to eliminate genomic DNA contamination. cDNA was synthesised from $2 \mu \mathrm{g}$ of RNA from each embryo sample with oligo-dT primers using the ImProm-II kit (Promega) according to manufacturer's instructions. Water was added in place of reverse transcriptase in control reactions. PCR was performed using Platinum Taq Polymerase (Invitrogen) and the primers listed in Table I. Cycle numbers for SpARC1-4 $(35,27,33,35$, respectively) and ubiquitin (27) were determined empirically such that the amplification was in the exponential phase. Amplified products were resolved on $1 \%$ agarose gels, stained with ethidium bromide $(5 \mu \mathrm{g} / \mathrm{ml})$
Table I. Sequence of primers used in this study

\begin{tabular}{ll}
\hline Primer & \multicolumn{1}{c}{ Sequence } \\
\hline SpARC1 1F & ATGGGCATCTACACCATATTCA \\
SpARC1 1R & TAGGGTAGTAGATATTGTATTCCAACC \\
SpARC2 1F & GCGATGTCAACTGTGATGCT \\
SpARC2 1F & CTCACAAGTGTTGCTGAGACC \\
SpARC3 1F & CACCGGATCCCCGCTGTATTCATCGTACCG \\
SpARC3 1R & ACCCTTTAGCGAACCCACAT \\
SpARC4 1F & ACAACTGTTCGGGTCTGTGG \\
SpARC4 1R & CAACTGTCGAACGTCGGA \\
SpUQ 1F & CCAGATGAACCCACTTCTTCCC \\
SpUQ 1R & TTTGCCCCTGCATCCCATCAACT \\
mCherry 1F & CACCATCGATTTATGGTGAGCAAGGGCGAG \\
mCherry 1R & TGAATTCAACTTGTACAGCTCGTCCATGC \\
\hline
\end{tabular}

and visualized on an UV transilluminator. Images were captured using Image Quant software (GE Healthcare).

ADP-ribosyl cyclase activity measurement. Frozen egg/embryo samples were thawed, homogenized in a buffer comprising $20 \mathrm{mM}$ HEPES ( $\mathrm{pH}$ 7.2) supplemented with complete ${ }^{\mathrm{TM}}$ EDTA free protease inhibitors and sonicated $(5 \times 5 \mathrm{~s})$ to form a $25 \% \mathrm{v} / \mathrm{v}$ homogenate. Homogenates were diluted 10 -fold in to a reaction mix containing a final concentration of either $20 \mathrm{mM}$ HEPES ( $\mathrm{pH}$ 7.2) and $1 \mathrm{mM}$ NAD (for cyclase activity measurements) or $50 \mathrm{mM}$ nicotinic acid (pH 4.8) and $1 \mathrm{mM}$ NADP (for base-exchange activity measurements). The reactions were allowed to proceed for up to $3 \mathrm{~h}$. To terminate the reactions, samples were diluted 10 -fold with water and heated at $60^{\circ} \mathrm{C}$ for $5 \mathrm{~min}$. Samples were then centrifuged for $3 \mathrm{~min}$ at $21000 \times \mathrm{g}$ to remove particulate matter. The products in the supernatants were resolved on an AG MP1 anion exchange HPLC column using a concave-up gradient of trifluoroacetic acid as described in (Churamani et al., 2007). Protein concentration was measured using the bicinchoninic acid protein assay kit (Pierce) and bovine serum albumin as the standard according to manufacturer's instructions.

Generation of SpARC4 mCherry. A construct encoding SpARC4 tagged with mCherry at its $\mathrm{N}$-terminus was generated by replacing the sequence of the myc tag of pCS2+ encoding N-terminally myc-tagged SpARC4 myc described in (Ramakrishnan et al., 2010) with that of mCherry. To achieve this, the coding sequence of mCherry was amplified by PCR using the primers listed in Table I and cloned into the ClaI and EcoRI sites of pCS2+ SpARC4 myc. Capped mRNA for SpARC4-mCherry was generated from the SP6 promoter of pCS2+ using the mMessage mMachine kit (Ambion).

Microinjection. Eggs were arranged in rows on dishes coated with protamine sulfate $(1 \% \mathrm{w} / \mathrm{v})$ in FSW supplemented with $4 \mathrm{mM}$ para amino benzoic acid (PABA). The eggs were fertilized and immediately microinjected with SpARC4 mRNA $(2 \mathrm{mg} / \mathrm{ml})$ according to standard protocols (Ettensohn et al., 2004). Following microinjection, the embryos were gently washed ten times with FSW to 
remove PABA and incubated in a humidified chamber at $12{ }^{\circ} \mathrm{C}$ for approximately $15 \mathrm{~h}$ before inspection.

Confocal microscopy. After hatching, the free-floating embryos were gently trapped on a clean glass slide using a cover slip and clay feet. Microscopy was performed at $12{ }^{\circ} \mathrm{C}$ using a Zeiss LSM 700 confocal microscope. Both bright field and DIC mode were used to capture images of whole embryos Fluorescence images of embryos expressing SpARC4-mCherry were captured upon excitation at $555 \mathrm{~nm}$.

\section{RESULTS AND DISCUSSION}

ADP-ribosyl cyclase expression is developmentally regulated. In previous studies, transcripts for SpARC2 (Churamani et al., 2008) and $\operatorname{SpARC} \alpha, \beta$ and $\gamma$ (Davis et al., 2008) were detected in sea urchin eggs. To extend these studies, we systematically analyzed the expression of all known SpARC isoforms including SpARC4 during early development by semi-quantitative PCR. Amplicons of the expected size for SpARC1 (972 bp), SpARC2 (638 bp) and SpARC3 (619 bp) were detectable in the egg (Fig. 1) in accord with earlier reports (Churamani et al., 2008; Davis et al., 2008). SpARC4 transcripts (expected amplicon size: 298 bp) were also detectable. The results indicate that all SpARCs are maternally expressed.

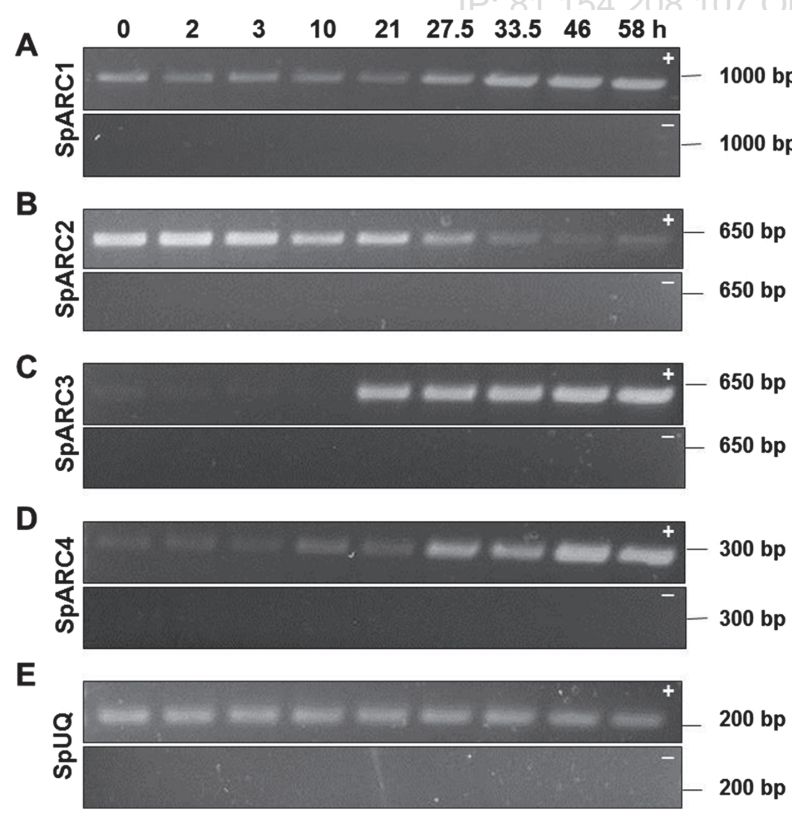

Figure 1. ADP-ribosyl cyclase expression is developmentally regulated. Semi-quantitative RT-PCR analysis for SpARC1 (A), SpARC2 (B), SpARC3 (C), SpARC4 (D) and ubiquitin (SpUQ, E). Analyses was performed using CDNA synthesised from egg and embryos at the indicated time post-fertilization either in the presence (top lanes; +) or absence (bottom lanes; -) of reverse transcriptase. Size of the molecular weight markers are indicated to the right. Results are representative of 3 independent fertilizations. Embryos were developed at $16{ }^{\circ} \mathrm{C}$.
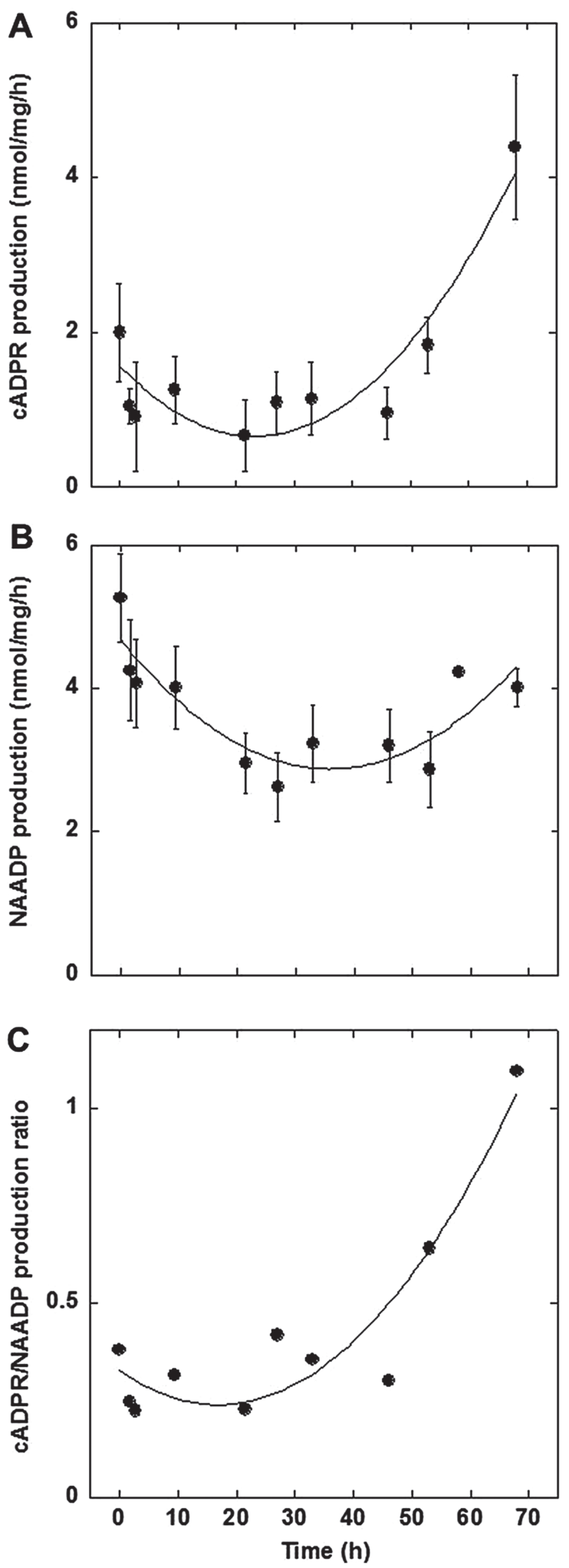

Figure 2. ADP-ribosyl cyclase activity is developmentally regulated. HPLC analyses of CADPR (A) and NAADP (B) production by egg and embryo homogenates prepared at the indicated time post-fertilization. (C) Ratio of cADPR:NAADP production. Data are presented as mean \pm S.E.M of 3 independent fertilizations. The embryos were developed at $16{ }^{\circ} \mathrm{C}$. 
SpARC expression showed marked changes during subsequent development (up to $58 \mathrm{~h}$ ). Transcript levels for SpARC1 were low in eggs and early embryos but increased from $27.5 \mathrm{~h}$ post-fertilization corresponding to the gastrula transition (Fig. 1(A)). Conversely, SpARC2 levels were relatively high in eggs and early embryos but decreased during the gastrula transition (Fig. 1(C)). For SpARC3, the transcript levels in the egg were low, decreasing further to almost negligible levels by $10 \mathrm{~h}$ (Fig. 1(C)). But at $21 \mathrm{~h}$ (corresponding to late blastula stage), SpARC3 expression increased (Fig. 1(C)). Finally, for SpARC4, the expression was low in the egg and early embryos but increased

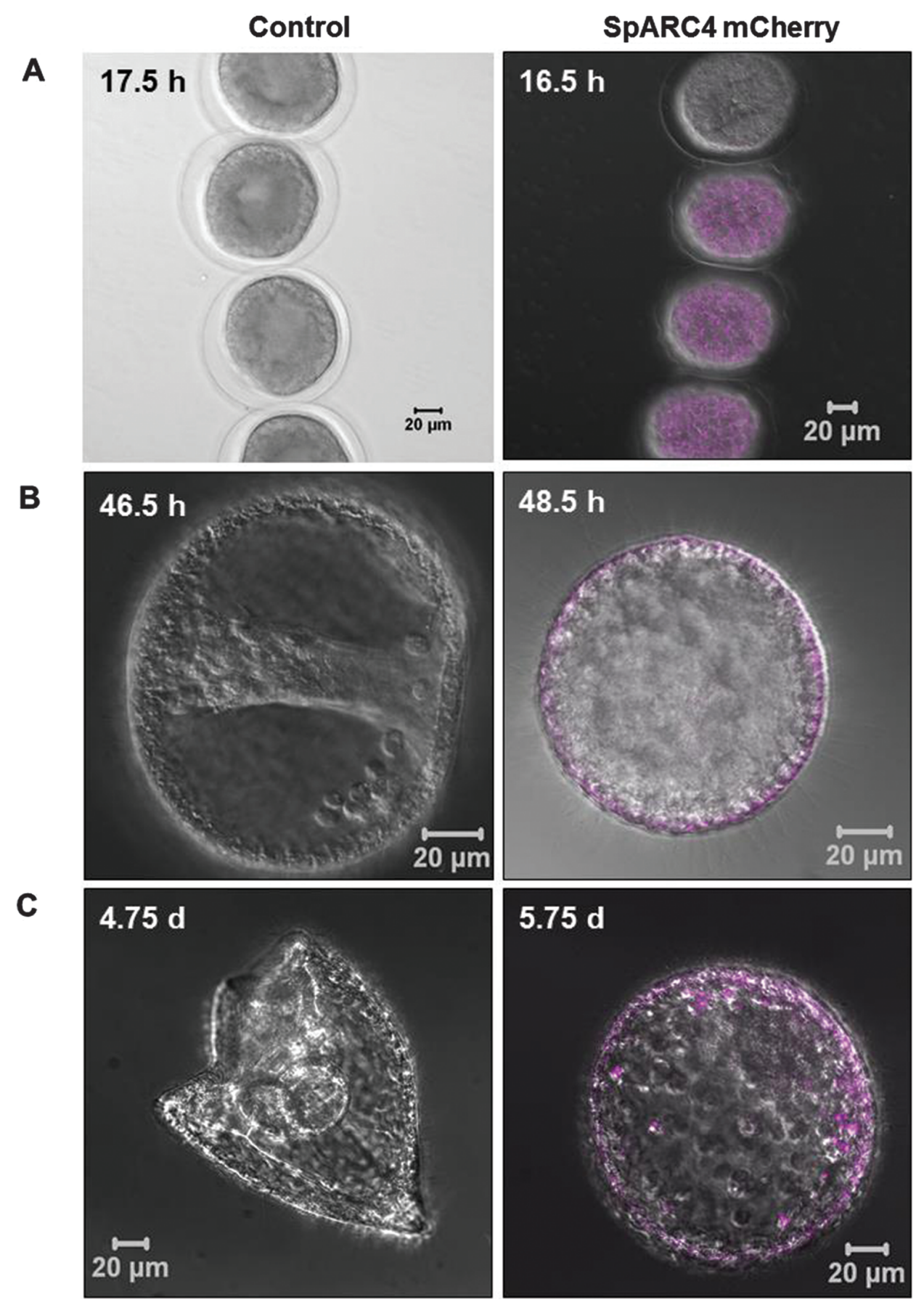

Figure 3. Over-expression of SpARC4 disrupts gastrulation. Morphology of control embryos (left) or embryos injected with SpARC4mCherry mRNA (right) at the indicated time post-fertilisation corresponding to blastula (A), gastrula (B) and prism/pluteus (C) stages. Images on the right are overlaid with confocal images of mRFP. Results are representative of 3 independent fertilizations. Embryos were developed at $12{ }^{\circ} \mathrm{C}$. 
significantly from $27.5 \mathrm{~h}$ post-fertilization, again corresponding to the gastrula transition (Fig. 1(D)).

Taken together, these results suggest that expression of each SpARC isoform is uniquely regulated during sea urchin embryogenesis. The presence of SpARC1 transcripts in embryos potentially reconciles previous discrepancies regarding the subcellular location of SpARC1/ $\beta$. Davis et al concluded that $\operatorname{SpARC} \beta$ is located within cortical granules in eggs (Davis et al., 2008) but these structures undergo exocytosis at fertilisation and thus are absent in somatic cells. This isoform must therefore reside elsewhere during later development possibly in the ER as reported for SpARC1 by Churamani et al. (Churamani et al., 2007).

ADP-ribosyl cyclase activity is developmentally regulated. Previous studies have identified ADP-ribosyl cyclase activities in the sea urchin egg (Graeff et al., 1998; Wilson and Galione, 1998). Little however is known regarding ADP-ribosyl cyclase activities in developing sea urchin embryos. We therefore measured endogenous cyclisation activity by HPLC using NAD as a substrate. cADPR production was observed at all the developmental time points tested (Fig. 2(A)). Cyclisation activity was $2 \pm 0.6 \mathrm{nmol} /$ $\mathrm{mg} / \mathrm{h}$ in the egg. Activity decreased during subsequent development reaching a minimum by $\sim 20 \mathrm{~h}$. Activity then recovered to that measured in the egg by $\sim 50 \mathrm{~h}$ (advanced prism stages) and further increased at $\sim 70 \mathrm{~h}$ (pluteus stage). At this point the production of CADPR was approximately two-fold higher than in eggs (Fig. 2(A)).

Base-exchange activity was also measured in parallel. For these experiments, homogenates were incubated with NADP and nicotinic acid at acidic $\mathrm{pH}$ to favour NAADP production. Like cADPR, NAADP was produced at all the developmental time points analysed (Fig. 2(B)). Baseexchange activity in the egg $(5.3 \pm 0.6 \mathrm{nmol} / \mathrm{mg} / \mathrm{h})$ was higher than cyclisation activity. Activity then decreased reaching a minimum at the blastula stage $(27 \mathrm{~h})$ and remaining relatively constant through gastrula (33 h), prism $(46 \mathrm{~h})$, and the advanced prism (53 h) stages. NAADP production rebounded at the pluteus stage (68 h) but remained lower than that in eggs (Fig. 2(B)).

cADPR and NAADP production are therefore differentially regulated during embryogenesis. This is highlighted in Figure 2(C) where the ratio of cyclisation and base-exchange is calculated at each developmental time point. The analysis shows that in eggs and early embryos, NAADP production is favoured (ratio $<1$ ) whereas later in development NAADP and cADPR production are more comparable (ratio $\sim 1$ ).

ADP-ribosyl cyclases regulate gastrulation. We have previously shown that recombinant SpARC isoforms have unique catalytic properties (Churamani et al., 2007; Churamani et al., 2008; Ramakrishnan et al., 2010). Notably, SpARC4 preferentially produces cADPR over NAADP (Ramakrishnan et al., 2010) whereas SpARC1 and SpARC2 show the opposite preference (Churamani et al., 2007; Churamani et al., 2008). The increase during early development of both the endogenous cyclisation:base exchange activity ratio (Fig. 2(C)) and expression of SpARC4 (Fig. 1(D)) prompted us to probe the role of SpARC4 in embryogenesis. To achieve this, development of embryos over-expressing SpARC4 was compared with control embryos from the same batch.

The control embryos developed as expected. Ciliated blastula stage embryos with intact fertilization envelopes were observed $15 \mathrm{~h}$ post-fertilization (Fig. 3(A)). The embryos hatched between 20-21 h. The free-swimming embryos initiated gastrulation after 46 h (Fig. 3(B)). The archenteron was fully developed and a pair of tri-radiate spicules were seen at the end of $3 \mathrm{~d}$. By $5 \mathrm{~d}$, the pluteus stage of the elongated larva with arms and tri-partitioned stomach was visible (Fig. 3(C)).

Confocal microscopy of embryos injected with mRNA encoding SpARC4 tagged with mCherry confirmed expression of the construct at $14 \mathrm{~h}$ post-fertilization. Expression persisted for at least 6 days of development. SpARC4-mCherry expressing embryos developed similarly to the control embryos up to the ciliated blastula stage (Fig. 3(A)). Importantly, most SpARC4-mCherry expressing embryos failed to gastrulate (Fig. 3(B)). Some embryos underwent delayed but abnormal gastrulation, such that the archenteron was curved and fused to the embryo wall at an abnormal angle with evidence of abnormal spicule formation (data not shown). These effects are likely specific to SpARCs, and unrelated to overexpression, since many proteins can be expressed in the embryo without deleterious consequences (for example see (Gokirmak et al., 2012)). Interestingly, a recent study showed that knockdown of sea star ADP-ribosyl cyclase caused lethality at the gastrula stage consistent with a conserved role for ADP-ribosyl cyclases in echinoderm development (Ramos et al., 2014). Thus, both overexpression and knockdown of ADP-ribosyl cyclases disrupts gastrulation pointing to a precise level of enzyme activity for sustaining echinoderm development.

\section{CONCLUSION}

In summary, we provide an analysis of ADP-ribosyl cyclase expression, activity and function during sea urchin development. Our results identify a potential role for ADPribosyl cyclases during gastrulation. $\mathrm{Ca}^{2+}$ signalling during this critical period has been previously described in vertebrate embryos (Webb and Miller, 2006). Sea urchins are unique in their expanded ADP-ribosyl cyclase complement even within echinoderms given that sea stars appear to possess only a single ADP-ribosyl cyclase (Ramos et al., 2014). Both cADPR and NAADP contribute to $\mathrm{Ca}^{2+}$ signals at fertilization of sea urchin eggs (Galione et al., 1993; Lee et al., 1993) which contrasts to mammalian 
eggs which are driven primarily by $\mathrm{IP}_{3}$ generated by a sperm-specific phosopholipase C (Saunders et al., 2003). We suggest that whilst the role of $\mathrm{Ca}^{2+}$ in regulating developmental events is likely conserved in deuterostomes, the underlying mechanisms for generating $\mathrm{Ca}^{2+}$ signals may differ. Further analysis of ADP-ribosyl cyclases during sea urchin development is thus warranted.

Acknowledgments: This work was supported by BBSRC grant BB/D018110/1 (to Sandip Patel), an MRC Ph.D. studentship (to Latha Ramakrishnan) and NIH grant HD058070 (to Amro Hamdoun). We thank the Bogue foundation (UCL) for additional financial assistance and Victor Vacquier for help and advice on the preparation of egg and embryo homogenates.

\section{REFERENCES}

Berridge. G., Cramer, R., Galione, A., and Patel, S. (2002). Metabolism of the novel $\mathrm{Ca}^{2+}$-mobilizing messenger nicotinic acid-adenine dinucleotide phosphate via a $2^{\prime}$-specific $\mathrm{Ca}^{2+}$-dependent phosphatase. Biochem. J. 365, 295-301.

Berridge, M. J., Lipp, P., and Bootman, M. D., (2000). The versatility and universality of calcium signalling. Nat. Rev. Mol. Cell Biol. 1, 11-21.

Churamani, D., Boulware, M. J., Geach, T. J., Martin, A. C., Moy, G. W., Su, Y. H., Vacquier, V. D., Marchant, J. S., Dale, L., and Patel, S. (2007). Molecular characterization of a novel intracellular ADP-ribosyl cyclase. PLOS ONE 2, e797.

Churamani, D., Boulware, M. J., Ramakrishnan, L., Geach, T. J., Martin, A. C., Vacquier, V. D., Marchant, J. S., Dale, L., and Patel, S. (2008). Molecular characterization of a novel cell surface ADP-ribosyl cyclase from the sea urchin. Cell Signal 20, 2347-2355.

Churamani, D., Geach, T. J., Ramakrishnan, L., Prideaux, N., Patel, S., and Dale, L. (2012). The signaling protein CD38 is essential for early embryonic development. J. Biol. Chem. 287, 6974-6978.

Clapper, D. L., Walseth, T. F., Dargie, P. J., and Lee, H. C. (1987). Pyridine nucleotide metabolites stimulate calcium release from sea urchin egg microsomes desensitized to inositol trisphosphate. J. Biol. Chem. 262, 9561-9568.

Davis, L. C., Morgan, A. J., Ruas, M., Wong, J. L., Graeff, R. M., Poustka, A. J., Lee, H. C., Wessel, G. M., Parrington, J., and Galione, A. (2008). $\mathrm{Ca}^{2+}$ signaling occurs via second messenger release from intraorganelle synthesis sites. Curr. Biol. 18, 1612-1618.

De Flora, A., Zocchi, E., Guida, L., Franco, L., and Bruzzone, S. (2004). Autocrine and paracrine calcium signaling by the CD38/NAD+/cyclic ADP-ribose system. Ann. NY Acad. Sci. 1028, 176-191.

Ettensohn, C. A., Wessel, G. M., and Wray, G. A. (2004). The invertebrate deuterostomes: an introduction to their phylogeny, reproduction, development, and genomics. Methods Cell Biol. 74, 1-13.

Galione, A., McDougall, A., Busa, W. B., Willmott, N., Gillot, I., and Whitaker, M. (1993). Redundant mechanisms of calcium-induced calcium release underlying calcium waves during fertilization of sea urchin eggs. Science 261, 348-352.

Gokirmak, T., Campanale, J. P., Shipp, L. E., Moy, G. W., Tao, H., and Hamdoun, A. (2012). Localization and substrate selectivity of sea urchin multidrug (MDR) efflux transporters. J. Biol. Chem. 287, 43876-43883.

Graeff, R., Liu, Q., Kriksunov, I. A., Hao, Q., and Lee, H. C. (2006). Acidic residues at the active sites of CD38 and ADP-ribosyl cyclase determine nicotinic acid adenine dinucleotide phosphate (NAADP) synthesis and hydrolysis activities. J. Biol. Chem. 281, 28951-28957.
Graeff, R. M., Franco, L., De Flora, A., and Lee, H. C. (1998). Cyclic GMP-dependent and -independent effects on the synthesis of the calcium messengers cyclic ADP-ribose and nicotinic acid adenine dinucleotide phosphate. J. Biol. Chem. 273, 118-125.

Howard, M., Grimaldi, J. C., Bazan, J. F., Lund, F. E., Santos-Argumedo, L., Parkhouse, R. M. E., Walseth, T. F., and Lee, H. C. (1993). Formation and hydrolysis of cyclic ADP-Ribose catalysed by lymphocyte antigen CD 38 Science 262, 1056-1059.

Jin, D., Liu, H. X., Hirai, H., Torashima, T., Nagai, T., Lopatina, O., Shnayder, N. A., Yamada, K., Noda, M., Seike, T., Fujita, K., Takasawa, S., Yokoyama, S., Koizumi, K., Shiraishi, Y., Tanaka, S., Hashii, M., Yoshihara, T., Higashida, K., Islam M. S., Yamada, N., Hayashi, K., Noguchi, N., Kato, I., Okamoto, H., Matsushima, A., Salmina, A., Munesue, T., Shimizu, N., Mochida, S., Asano, M., and Higashida, H., (2007). CD38 is critical for social behaviour by regulating oxytocin secretion. Nature 446, 41-45.

Kume, S., Muto, A., Inoue, T., Suga, K., Okano, H., and Mikoshiba, K. (1997). Role of inositol 1,4,5-trisphosphate receptor in ventral signaling in Xenopus embryos. Science 278, 1940-1943.

Lee, H. C. (2000). Enzymatic functions and structures of CD38 and homologs. Chem. Immunol. 75, 39-59.

Lee, H. C. (2012). Cyclic ADP-ribose and Nicotinic Acid Adenine Dinucleotide Phosphate (NAADP) as Messengers for Calcium Mobilization. J. Biol. Chem. 287, 31633-31640.

Lee H. C., Aarhus, R., and Walseth, T. F. (1993). Calcium mobilization by dual receptors during fertilization of sea urchin eggs. Science 261, 352-355.

Malavasi, F., Deaglio, S., Funaro, A., Ferrero, E., Horenstein A. L., Ortolan, E., Vaisitti, T., and Aydin, S. (2008). Evolution and function of the ADP ribosyl cyclase/CD38 gene family in physiology and pathology. Physiol. Rev. 88, 841-886.

Moreau, M., Neant, I., Webb, S. E., Miller, A. L., and Leclerc, C. (2008). Calcium signalling during neural induction in Xenopus laevis embryos. Philos. Trans. R Soc. Lond. B Biol. Sci. 363, 1371-1375.

Partida-Sanchez, S., Cockayne, D. A., Monard, S., Jacobson, E. L., Oppenheimer, N., Garvy, B., Kusser, K., Goodrich, S., Howard, M., Harmsen, A., Randall, T. D., and Lund, F. E. (2001). Cyclic ADP-ribose production by $\mathrm{CD} 38$ regulates intracellular calcium release, extracellular calcium influx and chemotaxis in neutrophils and is required for bacterial clearance in vivo. Nat. Med. 7, 1209-1216.

Patel, S. and Docampo, R. (2010). Acidic calcium stores open for business: Expanding the potential for intracellular $\mathrm{Ca}^{2+}$ signaling. Trends Cell Biol. 20, 277-286.

Perraud A. L., Fleig, A., Dunn C. A., Bagley L. A., Launay, P., Schmitz, C., Stokes A. J., Zhu, Q., Bessman M. J., Penner, R., Kinet J. P., and Scharenberg, A. M. (2001). ADP-ribose gating of the calciumpermeable LTRPC2 channel revealed by Nudix motif homology. Nature $411,595-599$.

Ramakrishnan, L., Muller-Steffner, H., Bosc, C., Vacquier, V. D., Schuber, F., Moutin, M. J., Dale, L., and Patel, S. (2010). A single residue in a novel ADP-ribosyl cyclase controls production of the calcium mobilizing messengers, cyclic ADP-ribose and nicotinic acid adenine dinucleotide phosphate. J. Biol. Chem. 285, 19900-19909.

Ramos, I., Reich, A., and Wessel, G. M. (2014). Two-pore channels function in calcium regulation. in sea star oocytes and embryos. Dev. 141, 4598-4609.

Saunders C. M., L.arman M. G., Parrington, J., Cox L. J., Royse, J., Blayney L. M., Swann. K., and Lai, F. A. (2003). PLC zeta: A spermspecific trigger of $\mathrm{Ca}^{2+}$ oscillations in eggs and embryo development. Dev. 129, 3533-3544. 
Schmid, F., Fliegert, R., Westphal, T., Bauche, A., and Guse, A. H (2012). Nicotinic acid adenine dinucleotide phosphate (NAADP) degradation by alkaline phosphatase. J. Biol. Chem. 287, 32525-32534.

Slusarski, D. C. and Pelegri, F. (2007). Calcium signaling in vertebrate embryonic patterning and morphogenesis. Dev. Biol. 307, 1-13.

Wallingford, J. B., Ewald, A. J., Harland, R. M., and Fraser, S. E. (2001)

Calcium signaling during convergent extension in Xenopus. Curr. Biol. $11,652-661$.

Webb, S. E. and Miller, A. L. (2006). $\mathrm{Ca}^{2+}$ signaling and early embryonic patterning during the blastula and gastrula periods of zebrafish and Xenopus development. Biochim Biophys Acta 1763, $1192-1208$.

Whitaker, M. (2006). Calcium at fertilization and in early development. Physiol. Rev. 86, 25-88.

Wilson, H. L. and Galione, A. (1998). Differential regulation of nicotinic acid-adenine dinucleotide phosphate and cADP-ribose production by cAMP and cGMP. Biochem J. 331, 837-843.

Zhao Y. J., Lam C. M., and Lee, H. C. (2012). The membranebound enzyme CD38 exists in two opposing orientations. Sci. Signal. 5 , ra67.

Received: 13 October 2016. Accepted: 27 February 2017. 